\title{
Reviewers of Current Issue
}

1. Prof. ASM Ashrafuzzaman

Professor

Department of Endocrinology, BIRDEM General Hospital, Dhaka, Bangladesh.

2. Prof. Tapash Kumar Moitra

Professor

Department of Surgery,

BIRDEM General Hospital,

Dhaka, Bangladesh.

3. Dr. Hashim Rabbi

Associate Professor

Department of HBPS,

BIRDEM General Hospital,

Dhaka, Bangladesh.

4. Dr. Mohammed Shahjahan Kabir

Associate Professor

Faculty of Medicine,

Quest International University,

Perak, Malaysia.

5. Dr. Wasim Md. Mohosin UI Haque

Associate Professor

Department of Nephrology,

BIRDEM General Hospital,

Dhaka, Bangladesh.
6. Dr. Chandra Shekhar Bala

Assistant Professor,

Department of Neurology,

NINS, Dhaka, Bangladesh.

7. Dr. Md. Abdul Baki

Assistant Professor

Department of Paediatrics, BIRDEM General Hospital, Dhaka, Bangladesh.

8. Dr. Md. Rashedul Isalam

Assistant Professor

Department of Neurology,

BIRDEM General Hospital,

Dhaka, Bangladesh.

9. Dr. Samira Rahat Afroze

Assistant Professor

Department of Internal Medicine, BIRDEM General Hospital,

Dhaka, Bangladesh.

10. Dr. Tabassum Samad

Assistant Professor

Department of Nephrology,

BIRDEM General Hospital,

Dhaka, Bangladesh. 